\title{
INJECTIVE COGENERATOR RINGS AND A THEOREM OF TACHIKAWA ${ }^{1}$
}

\author{
CARL FAITH
}

For Seth Camillo, and the happy parents.

\begin{abstract}
Tachikawa showed that a left perfect ring $R$ is an injective cogenerator in the category of all right $R$-modules iff there holds: (right FPF) every finitely generated faithful right module generates mod- $R$. In this paper, we simplify Tachikawa's long and difficult proof by first obtaining some new structure theorems for a general semiperfect right FPF ring $R$; the most important are: $R$ is a direct sum of uniform right ideals, and every nonzero right ideal of the basic ring $R_{0}$ of $R$ contains a nonzero ideal of $R_{0}$. Furthermore, if the Jacobson radical $\operatorname{rad} R$ is nil, then $R$ is right self-injective. Tachikawa's theorem is an immediate consequence. We also generalize a theorem of Osofsky on perfect PF rings to FPF rings.
\end{abstract}

1. Introduction. A ring $R$ is said to be right (F)PF provided that every (finitely generated) faithful right module $M$ generates the category $\bmod -R$ of all right $R$-modules. Theorems of Azumaya [66], Osofsky [66], and Utumi [67] characterize a right $\mathrm{PF}$ ring by the equivalent conditions.

$\left(\mathrm{PF}_{1}\right) R$ is right self-injective and semiperfect with essential right socle. (The socle is the largest semisimple submodule.)

$\left(\mathrm{PF}_{2}\right) R$ is right self-injective with finite essential right socle.

$\left(\mathrm{PF}_{3}\right) R$ is a finite direct sum, $R=\sum_{i=1}^{n} \oplus e_{i} R$, where $e_{i}^{2}=e_{i} \in R$ and $e_{i} R$ is a projective injective right ideal with simple socle, $i=1, \ldots, n$.

$\left(\mathrm{PF}_{4}\right) R$ is an injective cogenerator in mod- $R$.

$\left(\mathrm{PF}_{5}\right) R$ is right self-injective and every simple right module embeds in $R$.

(Compare Kato [68] and Onodera [68].)

These rings generalize the Quasi-Frobenius $(\mathrm{QF})$ rings of Nakayama (the Artinian PF rings), and the twosided PF rings of Morita [58]. The latter rings possess a duality between the reflexive right $R$-modules and the reflexive left $R$-modules induced by $\operatorname{Hom}_{R}(, R)$. A theorem of Tachikawa [69] establishes that any left perfect (in the sense of Bass [60]) right FPF ring $R$ is actually right PF; consequently right Artinian right or left FPF rings are QF. In this paper we generalize Tachikawa's theorem (in (2) of Theorem 1) and in doing so obtain a simpler (and self-contained) proof. (Most undefined terms are explained in $\$ 2$. Also, see Faith [76a], especially Chapter 24.)

1. THEOREM. (1) If $R$ is a semiperfect right FPF ring, then $R$ is a direct sum of uniform right prindecs (= principal indecomposable right ideals), and every

Received by the editors April 28, 1975 and, in revised form, December 29, 1975.

AMS (MOS) subject classifications (1970). Primary 16A36, 16A52; Secondary 16A49, 16A64.

'This article was written while the author held a Faculty Academic Leave from Rutgers University, 1973-74, and a visitor at the Institute for Advanced Study. 
nonzero right ideal of the basic ring $R_{0}$ of $R$ contains a nonzero ideal of $R_{0}$. (2) Moreover, if $\mathrm{rad} R$ is a nil ideal, then $R$ is right self-injective.

2. THEOREM (CONVERSE OF THEOREM 1). Any right self-injective semiperfect ring $R$ is right FPF if every nonzero right ideal of the basic ring $R_{0}$ contains an ideal of $R_{0}$.

Theorem 1 implies Tachikawa's theorem (via $\left.\mathrm{PF}_{1}\right)$ since any left perfect ring has nil radical and essential right socle (Bass [60]). Incidentally this proves

3. Corollary. Any right (or left) perfect right FPF ring is right self-injective.

We also prove

4. Theorem. A left FPF right $P F$ ring with nil radical is left $P F$.

5. Corollary. A left perfect left and right FPF right is $Q F$.

The corollary generalizes the theorem of Osofsky [66] for one-sided perfect two-sided PF rings. The corresponding question for one- or even two-sided perfect one-sided PF rings is open.

2. Background. Before going to the proofs of the stated theorem, we supply the relevant background material for these.

2.1 Definition AND Proposition. Let mod- $R$ denote the category of right $R$-modules for a ring $R$. An object $M$ of mod- $R$ is a generator iff the equivalent conditions hold:

G1. The set-valued functor $\operatorname{Hom}_{R}(M$,$) is faithful.$

G2. Given an object $X$ of mod- $R$, there is an index set $I$ and an exact sequence $M^{(I)} \rightarrow X \rightarrow 0$, where $M^{(I)}$ is the coproduct of I copies of $M$.

G3. There is a finite integer $n>0$, an object $Y$ of mod-R, and an isomorphism $M^{n} \approx R \oplus Y$.

G4. The trace ideal trace ${ }_{R} M=\sum_{f_{\varepsilon} \operatorname{Hom}_{R}(M, R)} f(M)$ equals $R$.

2.2 Definition and Proposition (Morita). Let $R$-mod denote the left-right symmetry of mod- $R$. Two rings $A$ and $B$ are similar provided that the equivalent conditions hold:

S1. $\bmod -A \approx \bmod -B$.

S2. There exists a finitely generated projective generator $P$ of mod- $A$ such that $B \approx$ End $P_{A}$.

S3. $A-\bmod \approx B$-mod.

In the case $\mathrm{S} 2, \operatorname{Hom}_{A}(P$,$) induces an equivalence \bmod -A \approx \bmod -B$ and the left adjoint $\otimes_{B} P$ is the inverse equivalence. (The equivalence of S1-S3 is Morita's theorem [58]. Cf. Bass [68] or Faith [73, Theorem 4.29].)

A Morita invariant property of $A$ is a property defined for the category of rings which $A$ and any ring similar to $A$ possesses.

Semiperfect rings. Let $R=\bigoplus_{i=1}^{n} e_{i} R$ be a direct sum decomposition of $R$ into principal indecomposable right ideals $e_{1} R, \ldots, e_{n} R$. By definition, then, $e_{i}$ is an idempotent $\neq 0, e_{i} R e_{i}$ is a local ring, and $e_{i} R$ is an indecomposable right ideal, which we call a right prindec, for short, $i=1, \ldots, n$. By a theorem of Bass [60], a ring $R$ has such a decomposition if (and only if) $R$ is semiperfect in the sense that $R / \mathrm{rad} R$ is semisimple, or, as we say, $R$ is semilocal, and idempotents of $R$ lift modulo $\operatorname{rad} R$. 
Basic modules and rings. Now assume the notation above. Renumber idempotents if necessary so that $e_{1} R / e_{1} J, \ldots, e_{m} R / e_{m} J$ constitute the isomorphism classes of simple right modules. Thus, every simple module $\approx$ some $e_{i} R / e_{i} J$, with $i \leqslant m$, and $e_{i} R / e_{i} J \approx e_{k} R / e_{k} J$ iff $i=k$, for all $i$ and $k \leqslant m$. The right ideal $B=e_{1} R+\cdots+e_{m} R$ is called the basic right module of $R$, $e_{0}=e_{1}+\cdots+e_{m}$ is then called the basic idempotent, and $e_{0} R e_{0} \approx \operatorname{End} B_{R}$ is the basic ring of $R$. The basic module is unique up to isomorphism, and if $f_{0}$ is any other basic idempotent, there is a unit $x$ of $R$ such that $f_{0}=x e_{0} x^{-1}$. Furthermore, $B$ is the unique (up to isomorphism) minimal (finitely generated projective) generator of mod- $R$, and, in fact, if $G$ is any other generator, there is a module $X$ of mod- $R$, and an isomorphism $G \approx B \oplus X$. (This follows from the Krull-Schmidt theorem. See, e.g., Bass [68, p. 19, (3.5)].) Thus, if $M$ $=M_{1} \oplus \cdots \oplus M_{n}=A \oplus X$, for modules $M_{i}, A$, and $X, i=1, \ldots, n$, where End $A_{R}$ is a local ring, then, for some $j, A$ is a direct summand of $M_{j}$. Thus, if each $M_{i}$ is indecomposable, then $M_{j}=A$.

By the Morita theorem, every semiperfect ring $R$ is similar to its basic ring. The ring $R$ is said to be self-basic iff $R=B$. (This condition is right-left symmetric, inasmuch as $R$ is self-basic iff $R / \mathrm{rad} R$ is a finite product of fields.) The basic ring of a ring is self-basic. Categorical properties such as $R$ is right self-injective ( = finitely generated projective right modules are injective) are Morita invariant properties, and therefore hold for $R$ iff they hold for the basic ring of $R$.

The term uniform is used in Goldie's sense, namely, a right module (or right ideal) $U$ is uniform iff $I \cap K=0$ for two submodules $I$ and $K$ imply that $I=0$ or $K=0$. Equivalently, the injective hull $E=\hat{U}$ of $U$ is indecomposable. (In this case, $B=$ End $E_{R}$ is a local ring by a theorem of Utumi [56].)

3. Proofs of Theorems. We begin with:

6. Proof of Theorem 1 . Let $B$ be the basic right module, and let $B=e_{1} R \oplus \cdots \oplus e_{n} R$ be its decomposition into a direct sum of mutually nonisomorphic right prindecs (see $\S 2$ ). Suppose, for example, that $I \cap K=0$ for two submodules $I$ and $K$ of $e_{1} R$. Then, $M=e_{1} R / I \oplus e_{1} R / K \oplus\left(1-e_{1}\right) R$ is a faithful right module inasmuch as its annihilator ideal $Q$ annihilates $\left(1-e_{1}\right) R$ and $e_{1} R$, that is, $Q$ annihilates $B$, which is faithful. Since $M$ therefore generates mod- $R$, we have $X \in \bmod -R$ such that $M \approx R \oplus X$ $=e_{1} R \oplus\left(1-e_{1}\right) R \oplus X$. Inasmuch as $e_{1} R / A$ and $e_{2} R / B$ are indecomposable, and $e_{i} R, i=1, \ldots, n$, all have local endomorphism rings, then by the KrullSchmidt theorem cited above, necessarily $e_{1} R \approx e_{1} R / I$ or $e_{1} R / K$. In the first case $I$ splits via projectivity of $e_{1} R$, so that indecomposability of $e_{1} R$ implies that $I=e_{1} R$, in which case $K=e_{1} R \cap K=I \cap K=0$, or $I=0$. Thus $I$ $=0$ or $K=0$ (also in case $e_{1} R \approx e_{1} R / K$ ), proving uniformity of $e_{1} R$, and of $e_{i} R, i=2, \ldots, n$, by symmetry. Since every right prindec $e R$ is isomorphic to one of the $e_{i} R, i=1, \ldots, n$, then every right prindec of $R$ is uniform, as required.

To complete the proof of (1), we may assume $R$ is self-basic. The top of any right $R$-module $M$ is defined to be $M / M J$, where $J=\operatorname{rad} R$, which is a semiperfect ring in the largest semisimple factor module. If $I$ is a right ideal containing no ideals $\neq 0$, then the right module $R / I$ is faithful, hence 
generates mod- $R$. Since $R$ is self-basic, then $R / I \approx R \oplus X$ for some $X$ $\in \bmod -R$. Hence $\mid$ top $R / I|=|$ top $R|+|$ top $X \mid$. But top $R / I=R /(I+J)$, that is, $\mid$ top $R / I|\leqslant| \operatorname{top} R|=| R / J \mid$, and top $X=X / X J$. It follows that $I \subseteq J$, and $X=X J$. Since $X$ is finitely generalized, this implies $X=0$, that is, $R / I \approx R$, so $I$ splits. Since $I \subseteq J$, this implies $I=0$.

(2) In order to prove that $R$ is right self-injective, it suffices to prove that $R_{0}$ is. Moreover, $R_{0}$ is semiperfect with nil radical $e_{0} J e_{0}$, where $e_{0}$ is the basic idempotent. Hence assume that $R$ is self-basic.

Let $u$ be an arbitrary element of the injective hull $e_{1} R$ of $\widehat{e_{1}} R$, and let $U=u R+e_{1} R$. Then, $M=U+\left(1-e_{1}\right) R$ is a faithful and finitely generated module, hence generates mod- $R$, so that $M \approx R \oplus X$ for some $X \in \bmod -R$, that is,

$$
M=\left(u R+e_{1} R\right) \oplus e_{2} R \oplus \cdots \oplus e_{n} R \approx e_{1} R \oplus e_{2} R \oplus \cdots \oplus e_{n} R \oplus X .
$$

Now, inasmuch as $e_{i} R e_{i} \approx$ End $e_{i} R_{R}$ is a local ring, $i=1, \ldots, n$, and $u R+e_{1} R$ is indecomposable, the Krull-Schmidt theorem implies that $e_{1} R$ is isomorphic to a direct summand of one of the direct summands of $M$. But, since $R$ is self-basic, and $e_{i} R$ is indecomposable, then $e_{1} R$ is not a direct summand of $e_{i} R$ for $i \geqslant 2$, hence $e_{1} R$ is isomorphic to a direct summand at $U$. But $U$ is uniform (contained in the injective hull of the uniform module $\left.e_{1} R\right)$, so therefore $U=u R+e_{1} R \approx e_{1} R$. Hence $B=$ End $U_{R}$ is a local ring $\approx e_{1} R e_{1}$, and $Q=\operatorname{rad} B$ is a nil ideal $\approx e_{1} J e_{1}$. Therefore, since the endomorphism $f: U \rightarrow U$ induced by the isomorphism $U \rightarrow e_{1} R$ has zero kernel, then $f$ cannot lie in $Q$, that is, $f$ cannot be nilpotent. Thus, $f$ lies outside of $Q$, hence $f$ is a unit, so that $U=f(U)=e_{1} R$. Since this is true for all $u \in \widehat{e_{1} R}$, then certainly $e_{1} R=e_{1} R$, so $e_{1} R$ is injective. Similarly, $e_{i} R$ is injective, $i \geqslant 2$, and so then is $R$.

7. Comment. It can be shown (Faith [7]) that any right FPF ring is right bounded in the sense that every essential right ideal contains a nonzero ideal. (Expressed otherwise: a cyclic module $R / I$ is faithful only if $I$ is inessential.) This generalizes part of Theorem 1 to nonsemiperfect rings.

8. Proof of Theorem 2 . Let $M=x_{1} R+\cdots+x_{n} R$ be a finitely generated faithful module. As in the proof of Theorem 1, we may suppose that $R$ is selfbasic, and therefore, the right ideal $K=\bigcap_{i=1}^{n}$ ann $x_{i}$ is either 0 , or else contains an ideal $B \neq 0$. But then

$$
M B=\sum_{i=1}^{n} x_{i} R B=\sum_{i=1}^{n} x_{i} B=0
$$

contrary to the faithful hypothesis on $M$. Hence $\bigcap_{i=1}^{n}$ ann $x_{i}=0$, and therefore $R \hookrightarrow M^{n}$ canonically. Since $R$ is right self-injective, then $R$ splits in $M^{n}$, hence $M^{n} \approx R \oplus X$ for some $X \in \bmod -R$, so therefore, $M$ generates mod- $R$. This proves right FPF.

We require the next lemma for the proof of Theorem 4. For a subset $S$ of $R$, we let $S^{\perp}=\{r \in R \mid S r=0\}$, and ${ }^{\perp} S$ its left-right symmetry.

9. LEMMA. Let $R$ be a self-basic semiperfect ring in which ideals faithful on the right generate mod- $R$. Moreover, assume that an element $c$ of $R$ is right regular (in the sense that $c^{\perp}=0$ ) only if $c R=R$. Then every simple left $R$-module $V$ embeds in $R$. 
Proof. Let $V$ be a simple left $R$-module, and $P=\operatorname{ann}_{R} V$. Since $R$ is semiperfect, then $R / P$ is simple Artinian, and, since $R$ is self-basic, $R / P$ is a field. Thus, $V \approx R / P \hookrightarrow R$ iff there is an $x \in R$ with ${ }^{{ }} x=P$. Since $P$ is a maximal left ideal, this happens iff $P^{\perp} \neq 0$. Thus $V \nrightarrow R \Rightarrow P^{\perp}=0$, hence $P$ generates mod- $R$ by the hypothesis on ideals. Since $R$ is self-basic, then $P \approx R \oplus X$ for some $X \in \bmod -R$, and hence there exists $c \in P$ with $c^{\perp}=0$. By the hypothesis on right regular elements, then $P=R$, a contradiction. This proves what we wanted.

10. REMARKS. 1. Every right faithful ideal generates mod- $R$ if $R$ is right $P F$.

2. The hypothesis on $c$ holds if either $R$ is right self-injective, or left perfect.

1 is obvious from the definition, and in 2, the map $c R \rightarrow R$ sending $c \mapsto 1$ has an extension to an element $f \in$ End $R_{R}$, when $R_{R}$ is injective, and then $y=f(1)$ satisfies $y c=1$. Then $R$ semiperfect (in fact the nonexistence of infinite sets of orthogonal idempotents suffices for this) yields $c y=1$. Thus $c R=R$.

When $R$ is left perfect, then there exist an integer $n$ and an element $y \in R$ such that $c^{n+1} y=c^{n}$. (This by the d.c.c. on principal right ideals, e.g. on $\left\{c^{n} R\right\}_{n=1}^{\infty}$.) Then $c^{\perp}=0 \Rightarrow c y=1$ as before.

11. Corollary (Kato [67]). If $R$ is right PF then every simple left module embeds in $R$.

Proof. Right $P F \Rightarrow$ semiperfect. We may assume that $R$ is self-basic, and apply 9 , using 10 .

\section{Corollary (Kato [67]). A right PF ring is left PF iff left self-injective.}

PROof. Immediate from 11 and the left-right symmetry of $\left(\mathrm{PF}_{5}\right)$.

13. Proof of Theorem 4. Immediate from 12 and Theorem 1.

14. Proof of Corollary 5. Apply Tachikawa's theorem to get one-sided $\mathrm{PF}$, apply Theorem 4 to get two-sided PF, and then Osofsky's theorem yields QF (Osofsky [66]).

\section{REFERENCES}

[66] G. Azumaya, Completely faithful modules and self-injective rings, Nagoya Math. J. 27 (1966), 697-708. MR 35 \#4253.

[60] H. Bass, Finistic dimension and a homological generalization of semi-primary rings, Trans. Amer. Math. Soc. 95 (1960), 466-488. MR 28 \#1212.

[68] , Algebraic K-theory, Benjamin, New York, 1968. MR 40 \#2736.

[67] C. Faith and E. A. Walker, Direct-sum representations of injective modules, J. Algebra 5 (1967), 203-221. MR 34 \#7575.

[73] C. Faith, Algebra: Rings, modules and categories. I, Springer-Verlag, Berlin and New York, 1973.

[76a] _ Algebra II: Ring theory, Springer-Verlag, Berlin and New York, 1976.

[76b] - Characterizations of rings by faithful modules, Lecture Notes, Math. Dept., Technion, Haifa, Israel.

[58] E. Matlis, Injective modules over Noetherian rings, Pacific J. Math. 8 (1958), 511-528. MR 20 \#5800.

[58] K. Morita, Duality for modules and its applications to the theory of rings with minimum condition, Sci. Rep. Tokyo Kyoiku Diagaku Sect A 6 (1958), 83-142. MR 20 \#183. 
[67] T. Kato, Self-injective rings, Tôkoku Math. J. (2) 19 (1967), 485-495. MR 37 \#247.

[68] - Some generalizations of QF-rings, Proc. Japan Acad. 44 (1968), 114-119. MR 38

\#183.

[68] T. Onodera, Uber Kogeneratoren, Arch. Math. (Basel) 19 (1968), 402-410. MR 38 \#2170.

[66] B. L. Osofsky, A generalization of quasi-Frobenius rings, J. Algebra 4 (1966), 373-387. MR 34 \# 4305; erratum, 36 \#6443.

[69] H. Tachikawa, A generalization of quasi-Frobenius rings, Proc. Amer. Math. Soc. 20 (1969), 471-476. MR 38 \# 5849.

[56] Y. Utumi, On quotient rings, Osaka Math. J. 8 (1956), 1-18. MR 18, 7.

[67] -, Self-injective rings, J. Algebra 6 (1967), 56-64. MR 35 \#219.

Department of Mathematics, Rutgers University, New Brunswick, New Jersey 08903 